\title{
NUMERICAL MODELING AND EXPERIMENTAL INVESTIGATION OF THE BREADBOARD MODEL OF A NEAR-FIELD INTERFERENCE MICROSCOPE
}

\author{
Victor Belichenko ${ }^{\mathrm{a}}$, Andrey Zapasnoy, Aleksandr Mironchev \\ National Research Tomsk State University, 634050, Tomsk, Russia
}

\begin{abstract}
A circuit design for technology of near-field interference microwave microscopy using evanescent fields of several active radiators (probes) is suggested and substantiated. The feasibility of providing the spatial overlap of the evanescent fields of two probes representing expanding coaxial conical horns is demonstrated. It is shown that the structure of the electromagnetic field in the region of overlap can effectively be controlled by changing the phase difference of oscillations arriving at inputs of the probes. This technology can be used for diagnostics of liquid media comprising dielectric inhomogeneities, flaw detection, and hygroscopy.
\end{abstract}

\section{Introduction}

Recently more and more researchers direct their attention to investigation of possibilities of near-field microwave diagnostics [1-7]. Such diagnostics involves medical (detection of human tumor formations) and industrial applications (flaw detection and hygroscopy), but in both cases restriction is imposed on the depth of field penetration into the examined medium or object. The penetration depth is of the order of the near field zone at the maximum wavelength from the frequency band of a probing signal. This necessitates a rigid connection of the receiver and transmitter of the probing system, since the field scattered by the objects in the near field zone of such system must be fixed. For such unilateral technology of sensing, the choice of the signal frequency band, receiving antenna aperture area, and transceiving system scanning altitude is very important.

In this work, an alternative sensing technology of bilateral near-field sensing using overlapping evanescent (non-propagating) microwave fields generated by spatially distributed systems of radiators is considered. Previously we conducted theoretical research [8] showing the significant role of the interference of energy fluxes arising due to overlapping (superimposition) of near fields of several radiators. Some considerations concerning the method of control over these fluxes for sensing of media, materials, and objects were also presented.

${ }^{\mathrm{a} C}$ Corresponding author: bvp@mail.tsu.ru 


\section{The prototype and the technique of experimental research}

By virtue of the foregoing, attention here is focused on the one hand, on detailed numerical modeling aimed at working out the design of the most important microscope units - probes, and on the other hand, on experimental investigations of the possibilities of a new version of the near-field microscope. Computer modeling was performed using the software package CST Microwave Studio. The model probing system comprised two identical conical coaxial horns each $36 \mathrm{~cm}$ long facing each other; the diameters of the central and outer conductors in the horn aperture were 3 and $7 \mathrm{~cm}$, respectively, and the gap between the horns was $5 \mathrm{~cm}$. The horns were excited by waveguide ports with identical characteristics, but the phase of the signal of the second port was shifted relative to the signal of the first port. The electric and magnetic field distributions were calculated in the frequency band from $0.2 \mathrm{GHz}$ to $10 \mathrm{GHz}$.

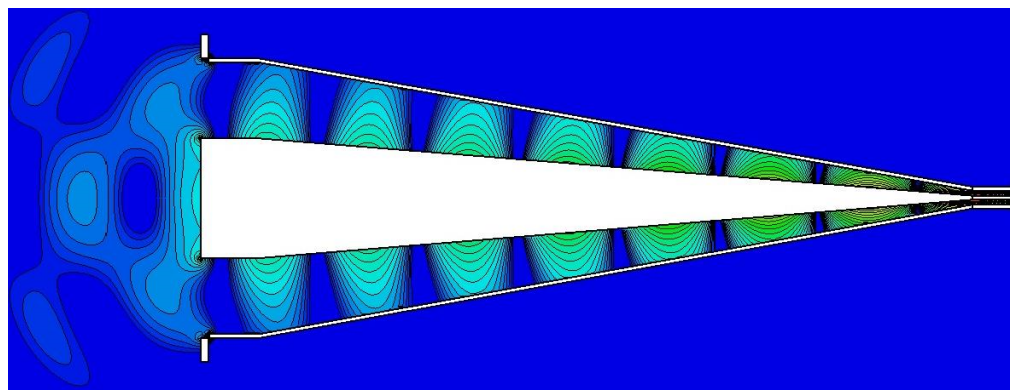

a)

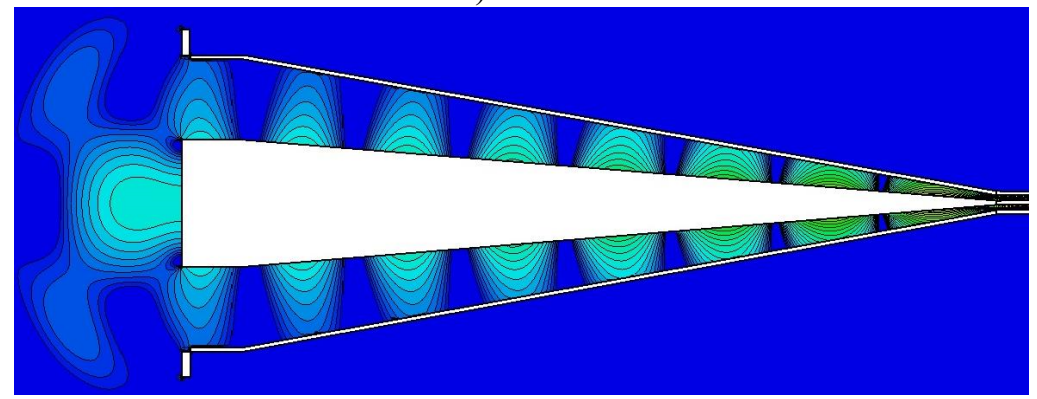

b)

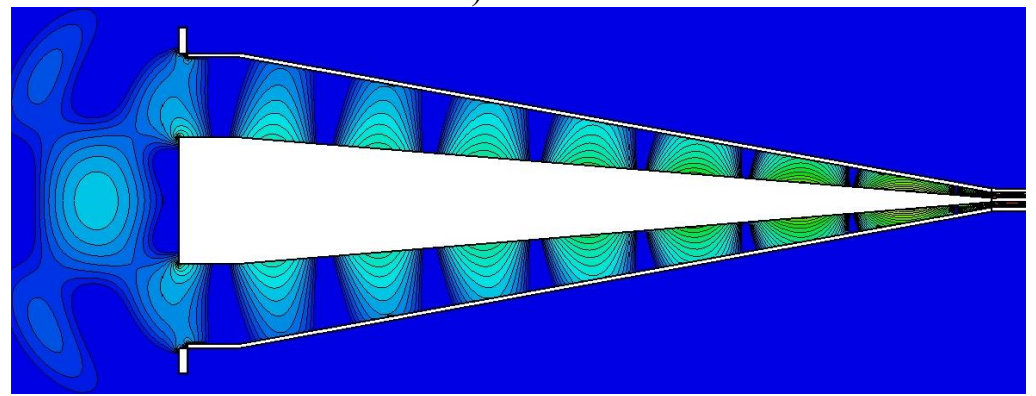

c)

Figure 1. Structure of the near field for the coaxial conical probe.

Figure 1 shows the distribution of the electromagnetic field with a frequency of $3 \mathrm{GHz}$ inside the single coaxial conical probe and near its aperture at the indicated time moments. At the initial moment of time (Figure 1, $a$ ) the region with high field concentration was localized mainly inside the probe, near its aperture. Figure 1, $b$ illustrates the moment of 
maximum field localization already outside of the probe. It can be seen that the field is localized near the end of the central conductor of the probe, and the sizes of the field localization region are of the order of the conductor diameter. Figure 1, $c$ shows the formation of the radiation field and the noticeable field attenuation near the end of the central conductor of the probe. The above-described special features of the field localization are in quite good agreement with the data presented in [9]. In particular, the conclusion that the spatial resolution of the probe should be of the order of the diameter of the inner conductor of the coaxial probe is qualitatively confirmed. We note that the frequency band used by us and the probe design allow the regime of object diagnostics using both evanescent and radiated waves to be realized.

The patterns of field distributions in the probing system modeled by two coaxial conical probes facing one another are shown below (Figure 2). By analogy with the single probe, modeling was performed for a frequency of $3 \mathrm{GHz}$. Its main purpose was to show the dynamics of forming the overlapping evanescent fields in the near-field zone of the probes and the dependence of their behavior on the initial phase difference $(\Delta \varphi)$ of the fields in the probes: a) $\Delta \varphi=0^{\circ}$, b) $\Delta \varphi=15^{\circ}$, c) $\Delta \varphi=30^{\circ}$, d) $\Delta \varphi=45^{\circ}$, and e) $\Delta \varphi=90^{\circ}$. It can be seen that by analogy with the case of the single probe, the TEM wave propagates in each probe. In the plane of the probe aperture, owing to a sharp break of its central conductor, the radiation fields start to form. In the immediate proximity to the ends of the central conductors of the probes, the near field is localized. It can be seen that for $0^{\circ}<\Delta \varphi<30^{\circ}$, the field localization differs significantly from that for the single probe: the near field occupies much larger spatial volume. Already for $\Delta \varphi=45^{\circ}$, the transformation of this volume starts to be manifested clearly, which is accompanied by predominant localization of the near field in the vicinity of the end of the central conductor of the left probe. Moreover, the highest degree of localization is observed at $\Delta \varphi=90^{\circ}$. The results of modeling presented here agree quite well with conclusions of work [8]. In this work, the theoretical investigations of the structure of the near field for several elementary radiating systems revealed the feasibility of efficient control over the interference parameters of energy fluxes formed in near overlapping fields of the radiators.

This feasibility has also been confirmed in the present work based on rigorous electrodynamic modeling of an actual probing system. It is important to emphasize that the parameters of the interference energy flux formed in the gap between the probe apertures can be controlled; moreover, they can be controlled electrically, namely, by changing the phase difference between the probe fields. This control is realized via "accentuating" one of the interference components of the Pointing vector. This circumstance means that a sufficiently small selected volume of the examined object or medium can be detected via flexible change of the regime of its irradiation by the evanescent fields created by a radiator system (moreover, the motionless system). 


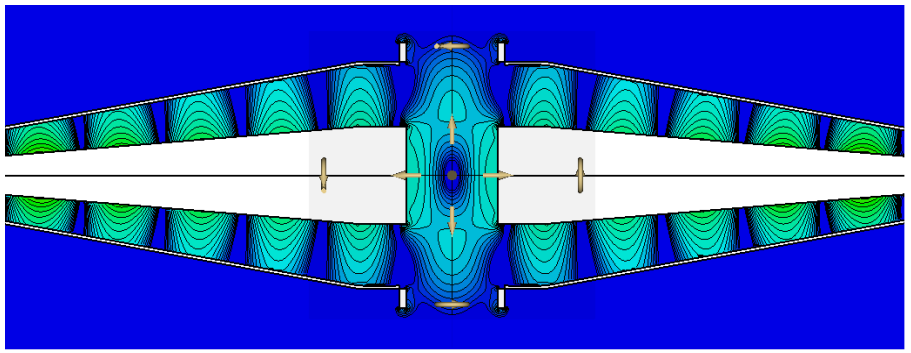

a) $\Delta \varphi=0^{\circ}$

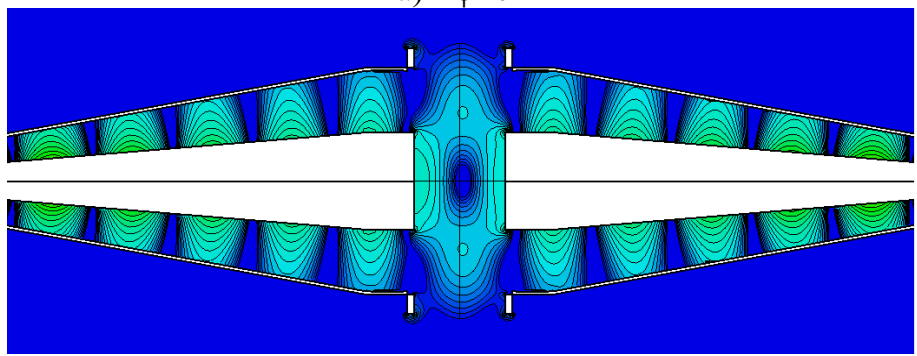

b) $\Delta \varphi=15^{\circ}$

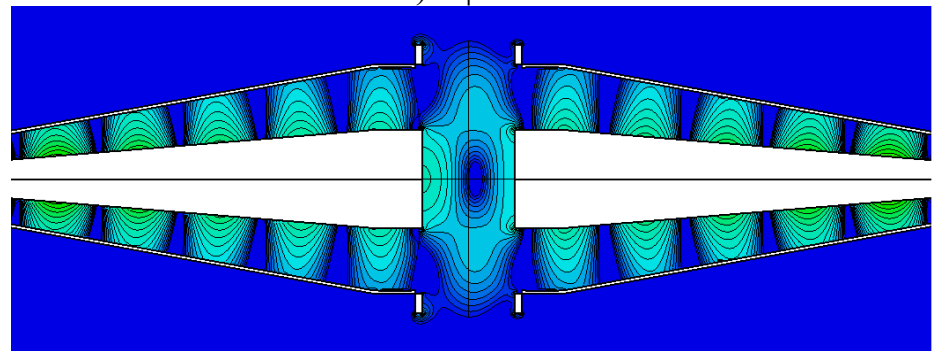

c) $\Delta \varphi=30^{\circ}$

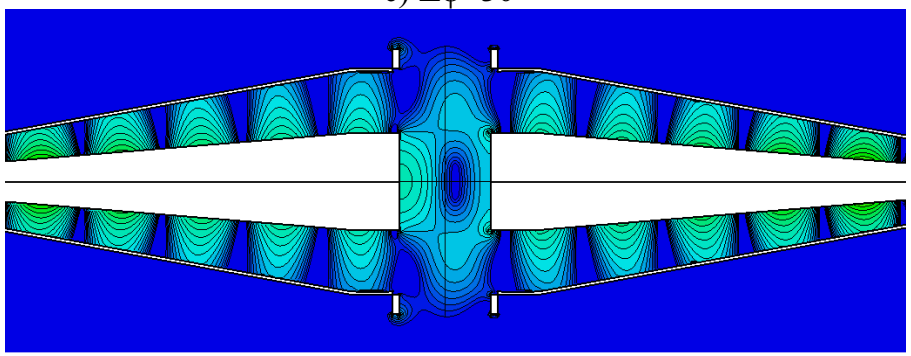

d) $\Delta \varphi=45^{\circ}$

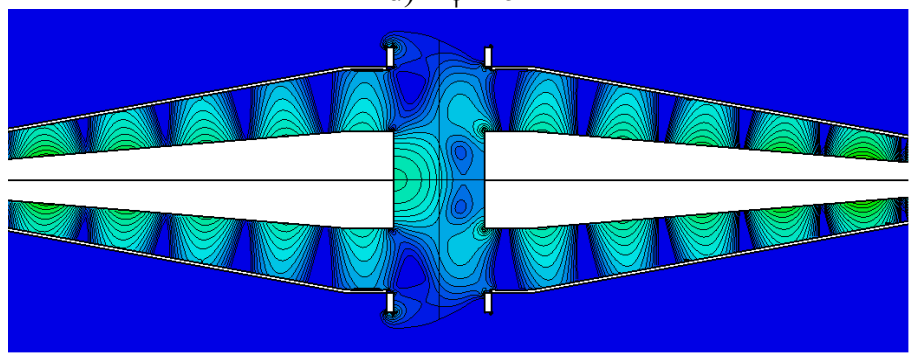

e) $\Delta \varphi=90^{\circ}$

Figure 2. Structure of the near field of two coaxial conical probes. 
The results of numerical modeling were used to propose a circuit design for the technology of near-field interference sensing simplified as much as possible. Based on this circuit design, a working breadboard model of the device was developed and tested experimentally to estimate its resolution and sensitivity.

\section{Experimental results: analysis and interpretation}

The breadboard model of the microwave interference microscope comprised the following main units: a N5230C PNA-L Network Analyzer (Agilent Technologies) with the working frequency band $10 \mathrm{MHz}-40 \mathrm{GHz}$, two coaxial conical horns (probes), power divider, and additional sections of transmission lines (Figure 3). The network analyzer allowed measurements to be performed with high speed and high accuracy, and the proper calibration provided correction for twelve error components.

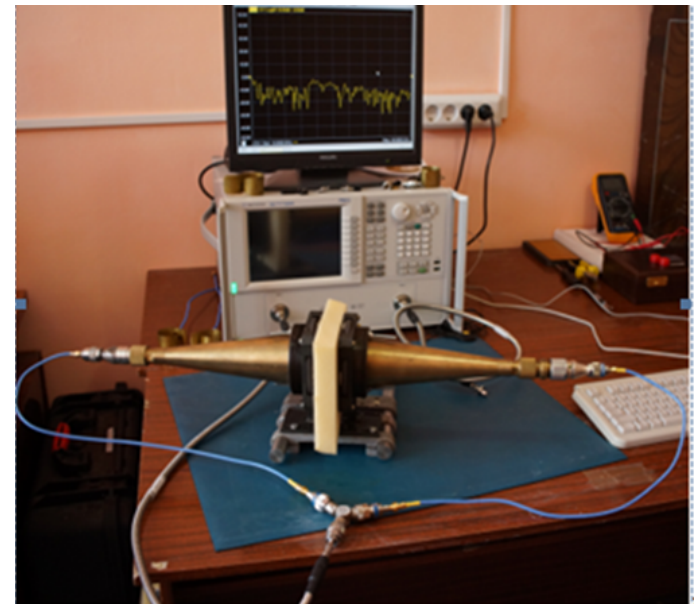

Figure 3. Breadboard model of the near-field interference microwave microscope.

For data registered by the network analyzer, the frequency dependence of the complex reflection coefficient (S11) of one of the probes was chosen. The frequency band during measurements was $500 \mathrm{MHz}-1 \mathrm{GHz}$. The digitization step was $250 \mathrm{kHz}$ since measurements in the chosen frequency band were performed in 2000 points. For convenience of perception, the initial dependences were normalized by the reflection coefficient of the objects without foreign components or defects. As objects of research, we had chosen a laminate board having defects in the form of through holes of different diameters, diesel fuel with different proportions of kerosene and synthetic engine oil impurities.

The first series of experiments was aimed at elucidation of the sensitivity of the breadboard model of the interference microwave microscope for diagnostics of the material quality in comparison with conventional systems of unilateral sensing. As an example, results of diagnostics of the laminate board having defects in the form of through holes 3,4 , and $10 \mathrm{~mm}$ in diameter (curves 1, 2, and 3 in Figure 4, a) are shown. To demonstrate the differences between the conventional sensing technology and the bilateral technology of near-field sensing by the overlapping evanescent microwaves, an experiment on diagnostics of defects of the laminate board using evanescent fields of only one probe was carried out (Figure $4, b$ ). Attention is drawn to the fact that the presence of a defect (even $10 \mathrm{~mm}$ in diameter, see curve 3 ) is very weakly manifested in the Figure (does not exceed $0.11 \mathrm{~dB}$ ). 


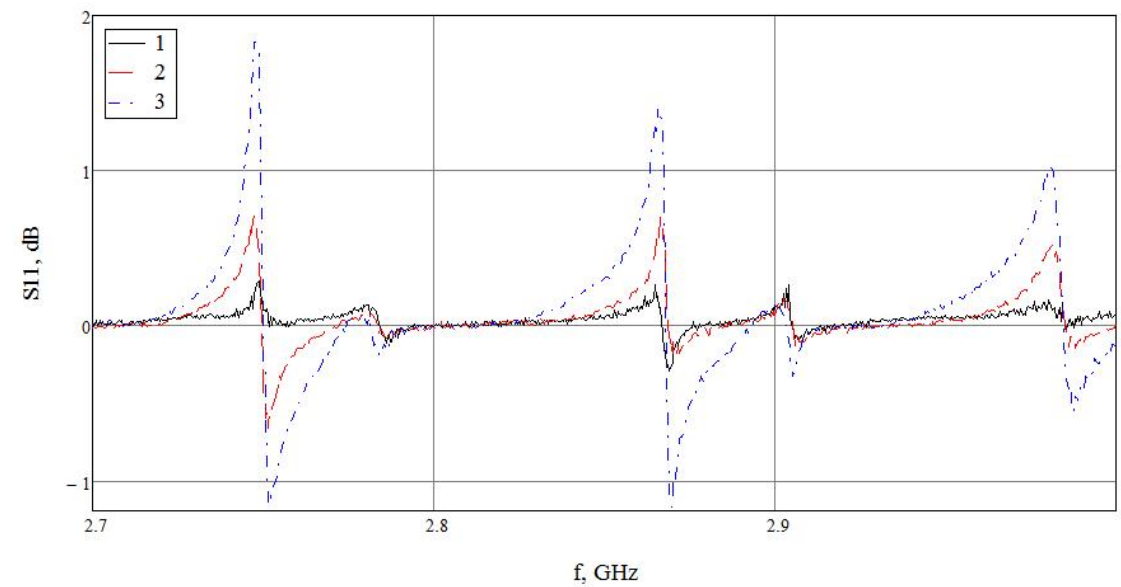

a)

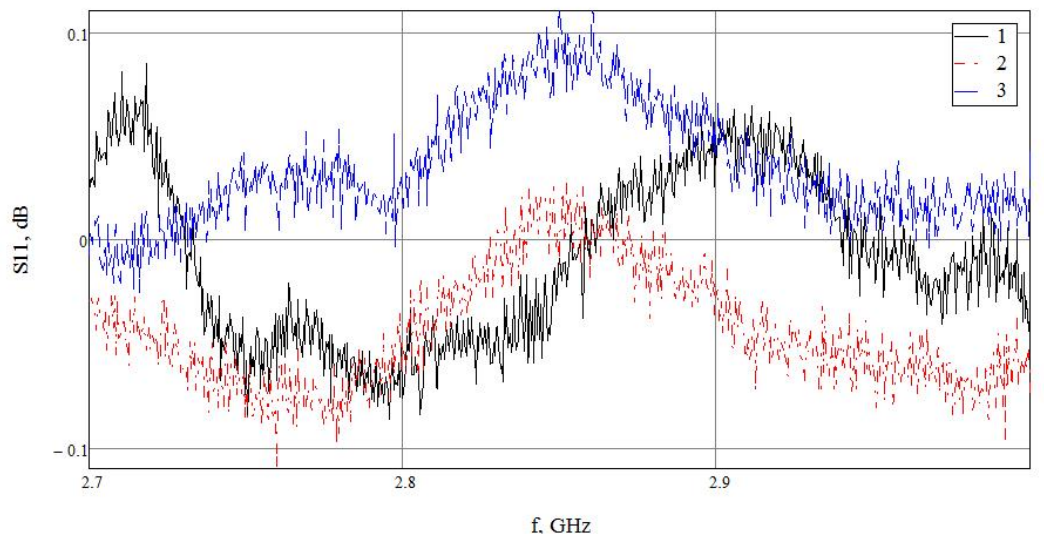

b)

Figure 4. Dependence of the coefficient of reflection from the laminate board $5 \mathrm{~mm}$ thick in the frequency band. The hole diameters were 3 (curve 1), 4 (curve 2), and $10 \mathrm{~mm}$ (curve 3).

Figure 5 shows the spectrum of the reflection coefficient for mixtures of diesel fuel and foreign impurity in the form of kerosene in proportions 10 and $20 \mathrm{ml}$ of kerosene in $200 \mathrm{ml}$ of diesel fuel (curves 2 and 3). Curve 1 here is for the diesel fuel without impurity. Curve 1 is shown to demonstrate changes arising in the signal after adding the impurity. The presence of a $10 \mathrm{ml}$ of the impurity is manifested sufficiently clearly at a number of frequencies, in particular, at frequencies of 0.77 and $0.89 \mathrm{GHz}$. The impurity added in double amount is quite reliably fixed not only at the above-indicated frequencies, but also at a lower frequency of $0.65 \mathrm{GHz}$. 


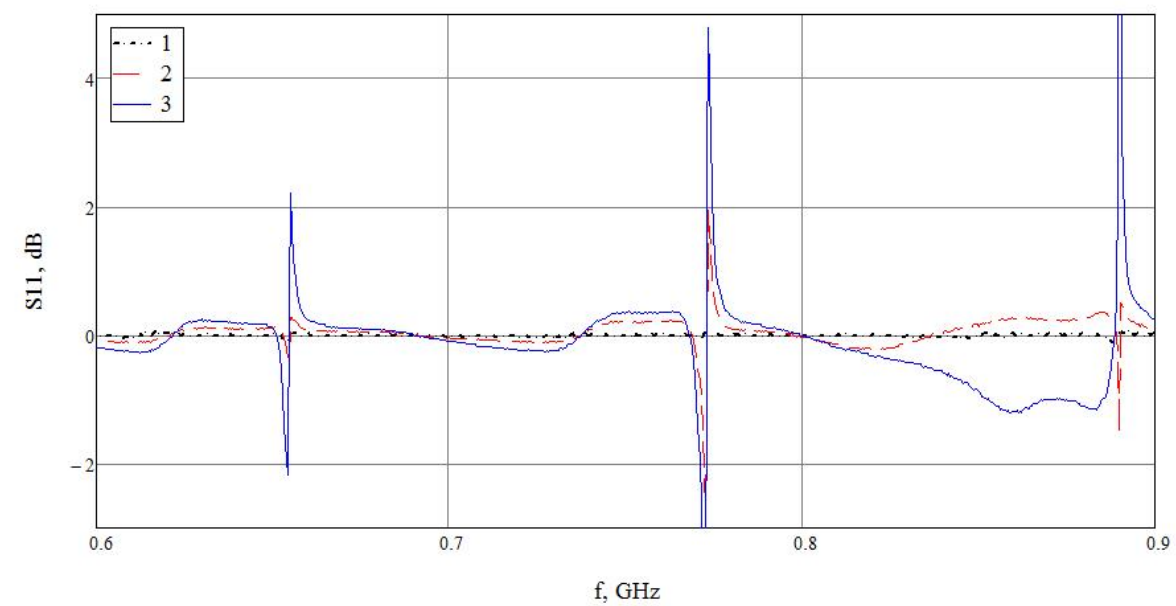

Figure 5. Frequency dependences of the reflection coefficient for mixtures of diesel fuel and kerosene.

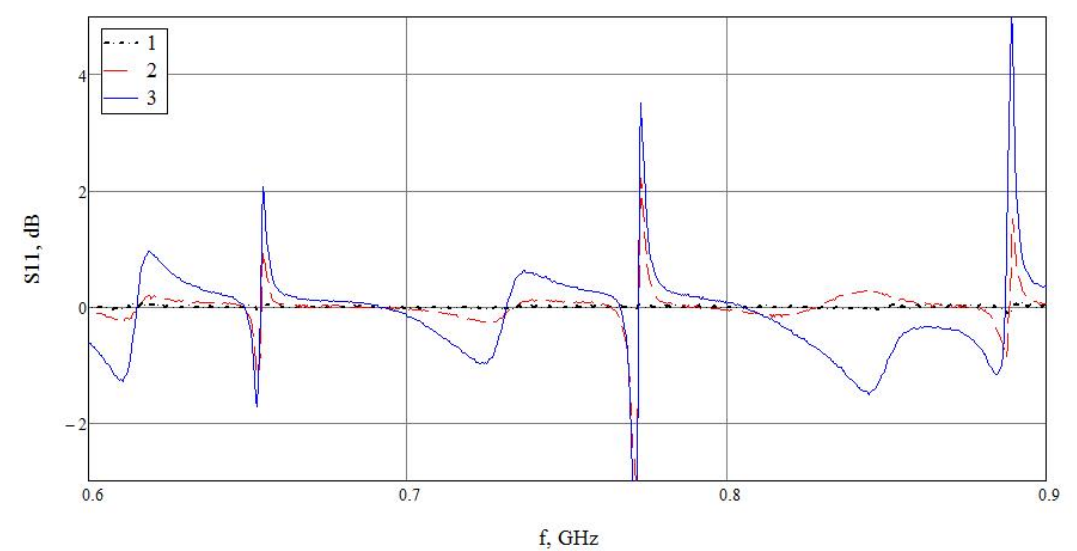

Figure 6. Frequency dependences of the reflection coefficients for mixtures of diesel fuel and oil.

The frequency dependences of the normalized reflection coefficient for mixtures of diesel fuel and synthetic engine oil are shown in Figure 6. Here curves 3 and 6 are for 10 and $20 \mathrm{ml}$ oil and $200 \mathrm{ml}$ diesel fuel, respectively; and curve 1 is for diesel fuel without impurity.

\section{Conclusion}

The plots shown here demonstrate the sensitivity of the breadboard model of the interference microwave microscope using overlapping counter-propagating evanescent fields of two coaxial conical probes. Numerical modeling performed by us demonstrated that the sensitivity of the microscope with one probe was much worse. This can be explained by lacking of the additional interference energy flux formed in the counterpropagating evanescent fields of two probes [8] and providing deeper penetration into the examined medium as well as by the increased sensitivity in comparison with conventional technologies of measurements $[10,11]$. 


\section{Acknowledgment}

This research carried out in 2016 was supported by «Russian Science Foundation» grant (No. 16-19-10272).

\section{References}

[1] M. Tabib-Azar, J.L. Katz, S.R. LeClair, IEEE Transactions on Instrumentation and Measurement 48, 1111 (1999) doi:10.1109/19.816123

[2] D.M. Hagl, D. Popovic, S.C. Hagness, J.H. Booske, M. Okoniewski, IEEE Transactions on Microwave Theory and Techniques 51, 1194 (2003) doi:10.1109/TMTT.2003.809626

[3] A.N. Reznik, N.V. Yurasova, Technical Physics 51, $86 \quad$ (2006) doi: $10.1134 / \mathrm{S} 1063784206010142$

[4] C. Gao, B. Hu, I. Takeuchi, K.-S. Chang, X.-D. Xiang, G. Wang, Measurement science and technology 16(1), 248 (2005) doi: 10-1088/0957-0233/16/1/033

[5] A.O. Oladipo, A. Lucibello, M. Kasper, S. Lavdas, G.M. Sardi, E. Proietti, F. Kienberger, R. Marcelli, N.C. Panoiu, Applied Physics Letters 105, 133112 (2014) doi: 10.1063/1.4897278

[6] K.P. Gaikovich, P.K. Gaikovich, Inverse Problems 26, 125013 (2010) doi: 10.1088/0266-5611/26/12/125013

[7] K.P. Gaikovich, Ye.S. Maksimovitch, Journal of radio electronics: electronic scientific journal, 1 (2015) [URL: http://jre.cplire.ru/jre/jan15/2/text.pdf (reference date of 14.06.2016)]

[8] V.P. Belichenko, A.S. Zapasnoy, P.V. Shestakov, Optics and Spectroscopy 118, 605 (2015) doi: 10.1134/S0030400X15040037

[9] S.M. Anlage, F.C. Wellstood, K. Vlahacos, D.E. Steinhauer, Near-field scanning microwave microscope having a transmission line with an open end Patent US No. 5900618, Int. Cl. ${ }^{6}$ G01J 1/20, Filed: Aug. 26, 1997, Publ.: May 4, 1999

[10] L. Cui, N. Marturi, E. Marchand, S. Dembélé, N. Piat, MATEC Web of Conferences 32, 05003 (2015) doi: 10.1051/matecconf/20153205003

[11] S.V. Kalinin, A. Gruverman, Scanning Probe Microscopy: Electrical and Electromechanical Phenomena at the Nanoscale (Volume 1), (New York: Springer, 2007) 